\title{
Syntactic means of positive emotions representation in American and British drama
}

\author{
Tatiana Nikolaevna Sineokova ${ }^{1 *}$, Svetlana Evgenyevna Rakhmankulova $^{1}$, and Ekaterina \\ Ivanovna Belyaeva ${ }^{1}$ \\ ${ }^{1}$ Nizhny Novgorod Linguistics University, Higher School of Translation and Interpreting, English \\ Language Department, Nizhny Novgorod, Russia
}

\begin{abstract}
The paper discusses the peculiarities of syntactic constructions presenting positive emotions in the speech of characters in dramas by modern American and British playwrights. The proposed research method suggests application of a correlative classification of extralinguistic and linguistic features including paradigmatic systems of emotional states and structural forms as an instrument for qualitative and quantitative analysis. Positive emotions are classified according to the criterion of their impact on speech-thought processes. A hierarchical paradigmatic classification of structural deformations of the emotionally neutral invariant including transformations, modifications and primary syntactic features is introduced. The prognostic potential of primary syntactic features in respect of the three types of emotional states is explored. It is shown that more than a half of primary features when used in isolation belong to the first (absolute) or the second level of dominancy according to the algorithmic identification of the emotional state type and that the character of positive emotions' effect on the speech-thought process (beneficial, boundary or destructive) correlates with the choice of syntactic structures. Differences in the number and types of syntactic constructions realized under the influence of positive and negative emotions are described. The role of qualitative and quantitative discrepancy in syntactic specificities with positive and negative emotions for further researches concerning identification issues purposes is shown.

Keywords: emotional speech, syntax, American and British drama.
\end{abstract}

\section{Introduction}

The ongoing interest in emotions' presentation in speech is manifested by various approaches, goals, methods of investigation and wide-ranging applications conditioned by further findings in related sciences [1,2]. Linguists study linguo-cultural peculiarities in emotional speech in different languages and among native and non-native speakers $[3,4]$. Special attention is also paid to differential features of emotionally coloured speech in speech genres [5] and on various levels of the language system including grammar [6, 7].

\footnotetext{
* Corresponding author: tatyana.sineokova@gmail.com
} 
Existing approaches to emotional syntax study face two major problems: the problem of choice of the emotional states that can be actually manifested by syntactic means and the problem of defining the method of systematization and description of linguistic elements.

Particular emotional states or groups of emotions formed in accordance with their quantitative and qualitative features (intensity, duration, positive or negative character, etc.) are investigated. A question arises why different emotions (for example, delight and anger) can be manifested by similar syntactic structures. An answer to this question may be the introduction of a new criterion when classifying the emotional sphere in syntactic research: consideration of the emotion's effect on the speech-thought process. Accordingly, three types of emotional influence can be singled out: 1) emotions have a beneficial impact; 2) emotions have a destructive impact, 3) the speaker tries to overcome an emotion's negative effect. Consequently, three types of emotional states are regarded: distress (destructive impact), eustress (beneficial impact) and boundary adaptation state [7].

A new approach to the syntactic classification is based on the notion that emotional speech is presented by deformed constructions in respect to the kernel structure regarded as an invariant. It includes a hierarchically structured paradigm including three levels of features' distinction: the level of transformations, the level of modifications and the level of primary features. This classification proved its prognostic potential for the emotional state detection [7]. Nevertheless, it seems useful to take a step back and consider the role of positive and negative emotions' impact on speech production within the proposed paradigmatic classification.

\section{Methods}

\subsection{Study Material}

The data analyzed in this paper is taken from 157 plays by modern American and British playwrights with the authors' stage directions concerning the emotional state of the speaker, both direct and indirect. Consequently, all the utterances can be easily referred to clusters expressing positive and negative emotions and, at the same time, to clusters reflecting one of the three above mentioned mechanisms of the emotion's impact on speech-thought processes.

\subsection{Instrumental Aspect}

The paradigmatic syntactic classification is adopted here as an instrument for analysis. Special attention is focused on two levels of the classification - the level of modifications and the level of primary features. Their correlation with the three types of emotional impact on speech is discussed. Identification of the emotional state type may be based on: a) primary syntactic features, b) their combinations and c) the number of primary syntactic features in the utterance. Not all primary syntactic features can serve for identification purposes but some of them (used in $53 \%$ of utterances in isolation) possess the prognostic potential. These syntactic features refer to three levels of dominancy in regard of the emotional state beginning with the first (absolute) up to the third level. 


\section{Findings and discussion}

\subsection{Modification repetition}

Modification repetition comprises 13 syntactic variants, each possessing some structural and semantic peculiarities. When we exclude negative emotions from the database under consideration and consider only positive ones, the number of primary features appears to be reduced to 7 .

Echolalic repetition is an iteration of the interlocutor's utterance fragment pronounced with the affirmative, interrogative or exclamatory intonation: Miriam: I've some chocolate biscuits today! - Alec (delighted like a child): Chocolate biscuits! [8: 167]. Its function is similar to that performed by interjections.

Articulatory perseverations reflect considerable changes in the work of organs of speech and are caused by operational level malfunction: Rosa (suddenly bursting into pure delight): Ha, ha, ha, ha, ha, ha! [9].

Repetition-vocative consists in an iteration of the interlocutor's name: Alec (jumping up in his delight): I can't believe it, Mummy! It ... Mummy ... [8: 188].

Tautological repetition is a full replication of words, word combinations or sentences: Mak: See the world. The world - the world ... is - is $-<\ldots>$ (Virtually in a trance.) [10: 61].

Reduced repetition is connected with the omission of the components of the initial fragment. We can clearly see it in the repetition-vocative example where the personal pronoun it is first realized within a clause, and then separately.

Expanded repetition is a repetition of the previous fragment in a wider clause: Lady (Rises ecstatic): Unpack the box! Unpack the box with the Christmas ornaments in it. [11].

Hybrid repetition consists in overlay of reduced and expanded repetition: Drishogue (awed): And there goes the scent of lavender again - no longer coy — but the scent of lavender a long time dead! [12: 62].

Echolalic repetition and articulatory perseverations have correlative links with the state of distress.

\subsection{Modification materially excessive elements}

Modification materially excessive elements (MEE) comprises 4 primary syntactic features, 2 of which were found with positive emotions.

MEE-affective with positive emotions contains interjections. MEE-vocative is the realization of the interlocutor's name. In the example below both features can be seen in one utterance: Mrs. Mayor (With a sharp sigh of joyful relief.) Oh, Robbie, I'm so glad! [13]. When used in isolation, none of these features belongs to any level of dominancy in respect to an emotional state.

\subsection{Modification breaks of the potentially united syntactic whole}

This modification includes 5 primary syntactic features, 4 of which were registered with positive emotions.

Break-emphasizer is the result of the speaker's intention to single out the most informative elements of the utterance: Lady (Exalted): I have - great - joy! [11].

Break-pausator may be caused by various reasons beginning with organs of speech malfunction up to difficulties in seeking words or forms. Within positive emotions its usage 
is restricted to the cases when the speaker is searching for appropriate words suiting the situation: Evans (tenderly): Ned told me — the secret — and I'm so happy, dear! [14: 146].

Clarifying break is conditioned by the speaker's intention to specify and complement the previous fragment. With positive emotions it is found within simple sentences and on the boundary between clauses: Robert (Slowly - his eyes shining): So we are going to be married - very soon — and I'm happy — and that's all there to say. [13].

Cut of a hypothetical syntactic whole is realized in the situations when affective processes deform speech-thought activity. The utterance remains unfinished, and it is possible only to guess what the speaker could say. The instances of its realization can be seen in examples with repetitions.

Break-emphasizer and clarifying break occupy the dominant level in respect to eustress, break-pausator belongs to the dominant level in respect to the boundary adaptation state, and cut of a hypothetical syntactic whole occupies the second level in respect of distress.

\subsection{Modification decipher constructions}

Whereas constructions described above (especially those with pauses) can be transformed into one sentence, decipher constructions consisting of synsemantic sentences cannot be united because of structural and semantic limitations. Out of 4 primary features constituting the modification 3 were found with positive emotions.

Introductory guide starts with the elementary identification of the image which first appears under the influence of affect, without clarifying its links with reality. The following sentence discloses its contents: Drishogue (delightedly): A Kelt! How well I knew some jewel of nature hid in how you looked and what you did and all you said! [12: 62].

Anticipation, on the contrary, is realized in cases when the emotional dominant is not an object (phenomenon) but its links with reality. At the beginning the object is presented in a general way, then its meaning is clarified, deciphered: Jennie $(<\ldots>$ excitedly $<\ldots>)$ : Isn't it marvellous? Dad being home? [8: 131].

Question-answer is a sequence of sentences when the speaker asks a question and answers it. The speaker's aim is to emphasize the theme by realizing it as a structurally independent component; the rheme is given in the following part: Curio (With unctuous excitement): See the balloon, great one? For the children. [10: 82]. The feature belongs to the dominant level of prognostic ones in respect of eustress.

\subsection{Modification transposition}

Syntactic transposition consists in breaking the rules of formal-functional reference of structures to definite classes. The modification includes 7 primary syntactic features, 4 of which were registered with positive emotions.

Improperly-affirmative sentences are manifested by rhetorical questions and by cliché constructions "interjection + if + pseudo subordinate clause in the negative form": Pearl (With childish excitement): Jees, Hickey, if you ain't a sport! [15].

Improperly-imperative sentences are presented by formally interrogative sentences: Michael (lifting his eyes skywards). Oh, d'ye here that! [12: 152].

Interjectional sentences and sentences-affectives contain interjections and proper (the interlocutor's) names like utterances with materially excessive elements.

Toning sentences are represented by different communicative and structural types of sentences realized with an exclamatory intonation. In many examples given above this feature is an additional one. Improperly-imperative sentences belong to the dominant level while improperly-affirmative sentences belong to the second one in respect of eustress. 


\subsection{Modification ellipsis}

The modification includes 2 primary syntactic features, 1 of which was registered with positive emotions.

Sentences-emphasizers do not contain the subject or the predicate representing the theme of the utterance. Thus, only emotional dominants serving as the rheme are realized: Barney (falling on his knees). Jayayus, a miracle! [12: 291]. This feature belongs to the dominant level in respect of eustress.

\subsection{Modification isolated constructions}

Isolated constructions are the result of realization of formally dependent constructions in isolation with an independent communicative status. It includes 2 primary syntactic features, 1 of which was found with positive emotions.

Potentially compound sentences start with the coordinative conjunctions 'and, or': Curio (With unctuous excitement): And there are refreshments, a trained kangaroo, two female acrobats, a male soprano! [10: 82]. This feature used alone does not possess a prognostic potential in respect of emotional states.

\subsection{Modification inversion}

The modification was not registered with positive emotions in the database under analysis.

\section{Conclusion}

The research shows that not all primary syntactic features and even modifications are manifested in speech realized under the influence of positive emotions. Out of 8 modifications $7(87.5 \%)$ were registered, and out of 40 primary features $22(55 \%)$ were found. Thus, constructive forms of negative and boundary emotions representation add significantly to the structural diversity of affective speech upon the whole. This difference in manifestations can be used in further researches concerning emotions' identification and speech synthesis.

Still the character of positive emotions' effect on the speech-thought process influences the choice of syntactic structures. Though not all primary syntactic features belonging to three levels of dominancy in respect of the type of emotional impact were found, a great number of those realized do possess prognostic potential (10 features out of $19 ; 52.6 \%) .3$ features serve as dominant ones in respect of distress, 1 feature - in respect of the adaptive state, and 6 features - in respect of eustress. The largest number of prognostically valuable features is realized within the modification breaks of the potentially united syntactic whole.

\section{Acknowledgments}

The reported study was funded by RFBR, project number 20-013-00361.

\section{References}

1. L. Alba-Juez, T. Larina, Russian Journal of Linguistics, 22(1), 9-37 (2018). https://doi.org/10.22363/2312-9182-2018-22-1-9-37 
2. Ch. Gillioz, J. R. J. Fontaine, C. Soriano, K. R. Scherer, Swiss Journal of Psychology, 75(3), 141-148 (2016). https://doi.org/10.1024/1421-0185/a000180

3. B. Ibragimova, A. K. Gizatullina, R. R. Mingazova, M. I. Olevskaya, Gênero \& Direito, 8(5) (2019). https://doi.org/10.22478/ufpb.2179-7137.2019v8n5.48637

4. J.-M. Dewaele, Journal of Pragmatics, 94, 112-127 (2016)

5. D. Zhgun, Litera, 4 (2019) https://doi.org/10.25136/2409-8698.2019.4.30344

6. A. Trotzke, Evolutionary Linguistic Theory, 1(1), 90-104 (2019). https://doi.org/10.1075/elt.00005.tro

7. T. N. Sineokova, Syntax of English emotional speech: A training manual (RuScience, Moscow, 2020)

8. C.W.A. Taylor, D. Pinner (eds.), New English Dramatists, 10, 157-222 (Penguin Books, London, 1967)

9. T. Williams, The Rose Tattoo (Dramatists Play Service, New York, 1998)

10. K. Cameron, Papp, in R. Schotter (ed.), The American place theatre: Plays, 31-88 (A Delta Original, New York, 1973)

11. T. Williams, Orpheus Descending (Dramatists Play Service, New York, 1987). Accessed on: November 12, 2020. [Online]. Available: http://openfist.org/attached\%20documents\%20(pdf)/Orpheus_Descending.TW.pdf

12. S. O’Casey, Collected Plays, Volume 4 (MacMillan \& Co, LTD, London, 1951)

13. E. O'Neill, Beyond the Horizon (The Project Gutenberg Ebook, 2018). Accessed on: November 12, 2020. [Online]. Available: http://www.gutenberg.org/files/58569/58569h/58569-h.htm

14. E. O'Neil, Three Plays of Eugene O’Neill's (Vintage Books, New York, 1958)

15. E. O'Neill, The Iceman Cometh ( Vintage Books, A Project Gutenberg of Australia, Sydney, 2004). Accessed on: December 23, 2020. [Online]. Available: http://gutenberg.net.au/ebooks04/0400021h.html 